\title{
$\mathrm{U}-\mathrm{Pb}$ датирование цирконов с помощью квадрупольного масс-спектрометра с индуктивно-связанной плазмой NexION 300S и приставки для лазерной абляции NWR 213
}

\author{
М.В. Зайцева ${ }^{1 *}$, А.А. Пупышев², Ю.В. Щапова ${ }^{12}$, С.Л. Вотяков \\ ${ }^{1}$ Иститут геологии и геохимии им. академика А.Н. Заварицкого УрО РАН, 620016, \\ Российская Федерация, Екатеринбург, ул. Академика Вонсовского, 15 \\ 2Уральский федеральный университет имени первого Президента России \\ Б.Н. Ельцина, 620002, Российская Федерация, Екатеринбург, ул. Мира, 19
}

*Адрес для переписки: Зайцева Мария Владимировна, E-mail: zaitseva.mv1991@gmail.com

Поступила в редакцию 15.11.16, после исправления - 23.11.16

Представлены оптимизированная процедура анализа изотопного состава $\mathrm{Pb}$ и U в цирконах, реализованная на квадрупольном масс-спектрометре с индуктивно-связанной плазмой NexION 300S (PerkinElmer) и приставке для лазерной абляции NWR 213 (ESI), и процедура определения изотопных отношений $\mathrm{Pb} / \mathrm{U}, \mathrm{Pb} / \mathrm{Th}$ и $\mathrm{Pb} / \mathrm{Pb}$ с последующим расчетом возраста цирконов. На примере цирконов Mud Tank и Plesovice изучено влияние операционных параметров лазерной приставки на величину элементного фракционирования. Показано различие значений данной характеристики для цирконов Mud Tank, 91500, GJ-1 и Plesovice. При оптимизированных параметрах приборов проведено сопоставление неопределенности измерения изотопных отношений с использованием многоколлекторного и квадрупольного масс-спектрометров. Выполнен сравнительный анализ возможности использования квадрупольного и многоколлекторного масс-спектрометров для U-Pb датирования цирконов с помощью лазерной абляции. С использованием квадрупольного масс-спектрометра проведены измерения и рассчитан возраст стандартных образцов циркона: Mud Tank 719

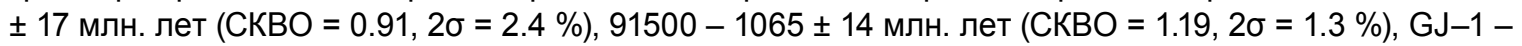
$600.8 \pm 8.8$ млн. лет (СКВО = 0.017, $2 \sigma=1.5 \%$ и и Plesovice $-336.6 \pm 7.7$ млн. лет (СКВО $=0.24,2 \sigma=$ $2.3 \%)$ ). Полученное значение возраста цирконов согласуется с литературными данными. Выполнены измерения и по конкордии рассчитан $\mathrm{U}-\mathrm{Pb}$ возраст акцессорного циркона из гранитоидов Новоусмановского массива (Урал) $-543 \pm 18$ млн. лет (СКВО $=2.7,2 \sigma=3.3 \%$ ), что удовлетворительно согласуется с данными SHRIMP.

Ключевые слова: масс-спектрометрия, лазерная абляция, датирование U-Pb, изотопные отношения, дискриминация ионов по массе, элементное фракционирование, цирконы

\section{Dating of zircons using NexION 3005 quadrupole mass spectrometer with inductively coupled plasma and NWR 213 attachment for laser ablation}

\author{
Maria V. Zaitceva ${ }^{1 *}$, Alexander A. Pupyshev ${ }^{2}$, Julia V. Shchapova ${ }^{1,2}$, Sergey L. \\ Votyakov ${ }^{1}$
}

${ }^{1}$ The Zavaritsky Institute of Geology and Geochemistry of the Ural Branch of the Russian Academy of Sciences (IGG UB RAS), ul. Akademika Vonsovskogo, 15, Ekaterinburg, 620016, Russian Federation

${ }^{2}$ Ural Federal University named after the first President of Russia B.N.Yeltsin), ul. Mira, 19, Ekaterinburg, 620002, Russian Federation

${ }^{*}$ Corresponding author: Maria V. Zaitceva, E-mail: zaitseva.mv1991@gmail.com 
A procedure of $\mathrm{U}-\mathrm{Pb}$ dating of zircons using the NexION 300S (PerkinElmer) quadrupole mass spectrometer with inductively coupled plasma and NWR213 (ESI) attachment for laser ablation has been developed. The optimum parameters for measuring $\mathrm{Pb} / \mathrm{U}, \mathrm{Pb} / \mathrm{Th}$, and $\mathrm{Pb} / \mathrm{Pb}$ isotopic ratios by NexION $300 \mathrm{~S}$ with NWR213 such as plasma costs, auxiliary gas flows Ar, carrier gas $\mathrm{He}$, power radiofrequency generation, and the temporal parameters of the signal registration have been obtained. The contribution of the elemental fractionation effect on the measurement results of standard natural zircons with various parameters of attachment for laser ablation has been examined. The analysis of the isotope ratios uncertainty measurement and the correction of mass bias have been performed. The developed scheme for determining the $\mathrm{Pb} / \mathrm{U}, \mathrm{Pb} /$ $\mathrm{Th}$ and $\mathrm{Pb} / \mathrm{Pb}$ isotope ratios of zircons with subsequent calculation of the age has been applied to the U-Pb dating of standard natural Mud Tank, 91500, GJ-1 and Plesovice zircons with differences in the degree of radiation damage. The age of the accessory zircon from the granitoid of the Novousmanovskii massif (Urals) has been calculated using the proposed data processing algorithm. A satisfactory agreement with earlier data has been shown. The comparative analysis between the use of quadrupole and multicollector mass spectrometers for the U-Pb dating of zircons by laser ablation has been performed.

Keywords: mass spectrometry, laser ablation, dating $\mathrm{U}-\mathrm{Pb}$, isotope ratios, mass bias, elemental fractionation, zircon

\section{Введение}

Задачи $\mathrm{U}-\mathrm{Pb}$ геохронологии сейчас достаточно успешно решаются методом масс-спектрометрии с индуктивно связанной плазмой (ИСП-МС) и лазерной абляцией проб (ЛА-ИСП-МС) [1] с использованием различных типов масс-спектрометров (МС) и приставок лазерной абляции (ЛА). Разработке методики датирования всегда предшествует сравнительный анализ литературных данных и выбор конкретной аппаратуры для реализации метода ЛА-ИСП-МС.

Первые коммерческие приборы ИСП-МС использовали квадрупольные масс-анализаторы (МА) [2]. Квадрупольные ИСП-МС превалируют в числе выпускаемых приборов данного метода и очень широко используются для решения разнообразных аналитических задач. Основными преимуществами квадрупольных МС являются [2, 3]: низкая стоимость, компактность, пониженные требования к вакуумной системе, высокая скорость накопления данных, широкий динамический диапазон, хорошая воспроизводимость результатов измерения.

Квадрупольные MC нашли широкое применение и для решения задачи U-Pb датирования цирконов методом ЛА-ИСП-МС [4-8]. Погрешность датирования для квадрупольных и многоколлекторных магнитно-секторных МС в большинстве случаев, не превышает 2 \% [4, 5, 9-12]. Применение квадрупольных МС, выполняющих измерения для различных изотопов последовательно, в общем случае, приводит к увеличению погрешности измерения изотопных отношений по сравнению с многоколлекторными приборами, которые, при надлежащей комплектации, реализуют принцип полностью одновременного измерения всех изотопов. Но при сопоставлении возможностей различных MC необходимо также учитывать влияние операционных параметров на погрешность датирования и меньшее время установления квадруполя для измерения сигналов различных изотопов по сравнению с установлением магнита (приборы с двойной фокусировкой).
Ранее нами была описана [1] отработка ЛА-ИСП-МС-методики определения изотопных отношений ${ }^{207} \mathrm{~Pb} /{ }^{206} \mathrm{~Pb},{ }^{206} \mathrm{~Pb} / 238 \mathrm{U},{ }^{207} \mathrm{~Pb} /{ }^{235} \mathrm{U}$ в минерале цирконе $\left(\mathrm{ZrSiO}_{4}\right)$ на многоколлекторном масс-спектрометре высокого разрешения Neptune Plus (ThermoScientific) с приставкой для ЛА NWR 213 (ESI). Приведен алгоритм обработки данных по определению изотопных отношений $\mathrm{Pb} / \mathrm{U}, \mathrm{Pb} / \mathrm{Th}$ и $P b / P b$, даны рекомендации по выбору стандартных образцов цирконов для датирования, оценено влияние операционных параметров приставки для ЛА на значение параметра элементного фракционирования, представлены результаты датирования стандартных цирконов. Показано, что основной трудностью реализации методики являлась невозможность одновременного измерения всех необходимых сигналов изотопов на имеющемся наборе коллекторов конкретного прибора: диапазон измерения масс ограничен $\pm 15 \%$ от центральной массы. Поэтому при переключении магнита МС с одной линии измерения на другую (время измерения одной линии $-0.066 \mathrm{c}$, время переключения магнита между линиями - 0.5 с) существенный объем материала образца, поступающего в плазму разряда при непрерывной ЛА, не детектируется, что может оказывать влияние на качество датировок цирконов.

Целью данной работы является отработка ЛА-ИСП-МС-методики определения изотопных отношений ${ }^{207} \mathrm{~Pb} /{ }^{206} \mathrm{~Pb},{ }^{206} \mathrm{~Pb} /{ }^{238} U$ и $^{207} \mathrm{~Pb} /{ }^{235} U$ в минерале цирконе $\left(\mathrm{ZrSiO}_{4}\right)$ на квадрупольном масс-спектрометре с лазерной приставкой для определения возраста цирконов

\section{1. Аппаратура и образцы для анализа}

Для U-Pb датирования использовали следующее оборудование: квадрупольный масс-спектрометр с индуктивно-связанной плазмой NexION $300 S$ (PerkinEImer) (диапазон анализируемых масс -5-285 а.е.м.; разрешающая способность - 0.3-3.0 а.е.м.; режим работы - стандартный); приставка NWR 213 (ESI) для лазерной абляции проб (лазер Nd:YAG: длина волны излучения 213 нм, длительность импульса 4-7 нс, частота следования 
импульсов 1-20 Гц, диаметр кратера 4-110 мкм; стандартная ЛА-ячейка объемом $100 \mathrm{~cm}^{3}$, эфрфективный объем ячейки $10 \mathrm{~cm}^{3}$ ), синхронизированная со спектрометром. Газы для оборудования МС и ЛА: Не (марка А, ТУ 0271-135-31323949-2005) для транспортировки материала пробы после абляции из ячейки приставки для ЛА и Ar жидкий (соответствует ГОСТ 10157-79) для транспортировки пробы в горелку МС. Определение значения ширины пика на половине его высоты (FWHM) в рамановских спектрах цирконов выполняли на спектрометpe Horiba LabRam HR800 Evolution (возбуждение Не-Ne лазером с длиной волны 633 нм, пространственное разрешение 1-2 мкм, спектральное разрешение $1 \mathrm{CM}^{-1}, T=300 \mathrm{~K}$ ).

В качестве образцов для анализа использовали природный циркон Plesovice ([13] из калиевого гранулита южной части массива Bohemian, Чехия; предоставлен Jiri Slama, University of Bergen, Norway) и образцы, уже изученные в [1]:

- стандартное синтетическое стекло NIST SRM 612 (Standard Reference Material of the National Institute of Standards and Technology, USA) [14] (сертифицированные содержания $\mathrm{Pb}, \mathrm{U}$ и Th составляют 38.57 , 37.38 и 37.79 ppm соответственно);

- природный циркон Mud Tank (из карбонатитов, Австралия [15]);

- природный циркон GJ-1 ([16], образец предоставлен Национальным Центром геохимической эволюции и металлогении континентов GEMOC Университета Маккуори, Сидней, Австралия);

- природный циркон 91500 (музейный образец из Kuehl Lake, Renfrew County, Ontario, Canada [17, 18]).

Данные по составу природных цирконов представлены в табл. 1. Перед анализом поверхность образцов, смонтированных в шашки из эпоксидной смолы, шлифовали, полировали и обрабатывали $0.5 \mathrm{H} \mathrm{HNO}_{3}$ (3 \% мас.) для удаления загрязнений.

\section{2. Операционные параметры измерения}

\section{1. Масс-спектрометр}

Во многих работах $[4-8,21]$ при измерении изотопных отношений наиболее часто используют следующие операционные параметры для квадрупольного ИСП-МС: высокочастотная мощность 1350-1450 Вт, плазмообразующий поток аргона 14-

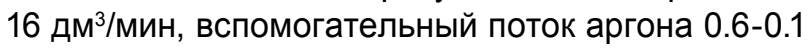

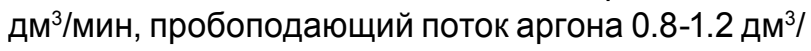
мин, измерение фона 10-20 с, время сбора полезного сигнала 30-60 с (в зависимости от количества измеряемых изотопов), в основном режим счета импульсов - прыжки по пикам (peak hopping), время пребывания на масce (dwell time) - 10-20 мс.

Для измерения сигналов от изотопов ${ }^{202} \mathrm{Hg}$, ${ }^{204} \mathrm{~Pb},{ }^{206} \mathrm{~Pb},{ }^{207} \mathrm{~Pb},{ }^{208} \mathrm{~Pb},{ }^{238} \mathrm{U}$ и ${ }^{232} \mathrm{Th}$ (выбор данных изотопов охарактеризован в работе [1]) была применена стандартная схема сканирования по массам в режиме «прыжки по пикам». Сигналы, полученные в одном измерении, составляют цикл реплик (replicate). В одной реплике можно задавать несколько циклов сканирования (sweeps) всего диапазона. Использовали для измерения время пребывания на каждой массе изотопа - 10 или 15 мс (см. табл. 2), количество циклов сканирования -2 , количество реплик - 700. В этих условиях время измерения одной реплики составляет $182 \mathrm{Mc}$, а общее время измерения МC - 127.960 с (время работы лазера при этом - 50 с). При измерении изотопных отношений Pb в стекле NIST SRM 612 кор-

Таблица 1

Содержание U, $\mathrm{Pb}$ и Th и значения изотопных отношений в цирконах

Table 1

$\mathrm{U}, \mathrm{Pb}$ and Th values and isotope ratios in zircons

\begin{tabular}{|c|c|c|c|c|c|c|c|c|}
\hline $\begin{array}{l}\text { Цир- } \\
\text { кон }\end{array}$ & $\begin{array}{c}\text { Возраст, млн. } \\
\text { лет }\end{array}$ & $\mathrm{U}, \mathrm{ppm}$ & $\begin{array}{l}\mathrm{Pb}, \\
\mathrm{ppm}\end{array}$ & $\begin{array}{l}\text { Th, } \\
\mathrm{ppm}\end{array}$ & $\begin{array}{c}{ }^{206} \mathrm{~Pb} /{ }^{238} \mathrm{U} \\
(2 \sigma)\end{array}$ & $\begin{array}{c}{ }^{207} \mathrm{~Pb} / /^{235} U \\
(2 \sigma)\end{array}$ & $\begin{array}{c}{ }^{207} \mathrm{~Pb} /{ }^{206} \mathrm{~Pb} \\
(2 \sigma)\end{array}$ & $\begin{array}{c}\text { Метод опре- } \\
\text { деления }\end{array}$ \\
\hline \multirow[t]{2}{*}{$\begin{array}{l}\text { Mud } \\
\text { Tank }\end{array}$} & $732 \pm 5^{(1)}$ & $\begin{array}{r}6.1- \\
36.5 \\
\end{array}$ & $\begin{array}{l}0.73- \\
4.39 \\
\end{array}$ & - & - & - & - & $\begin{array}{c}\text { ЛА-ИСП-МС } \\
{[15]}\end{array}$ \\
\hline & $727 \pm 3^{(1)}$ & 29 & 4 & 116 & $\begin{array}{c}0.11921 \pm \\
0.00286\end{array}$ & $\begin{array}{c}1.0463 \pm \\
0.0586\end{array}$ & $\begin{array}{c}0.06366 \pm \\
0.00324\end{array}$ & $\begin{array}{c}\text { ЛА-ИСП-МС } \\
\text { [19] }\end{array}$ \\
\hline 91500 & $\begin{array}{l}1062.4 \pm 0.4^{(2)} \\
1063.5 \pm 0.5^{(3)}\end{array}$ & $55-82$ & $13-17$ & $\begin{array}{c}28.61 \pm \\
0.07\end{array}$ & $\begin{array}{c}0.17917 \pm \\
0.00016\end{array}$ & $\begin{array}{c}1.8502 \pm \\
0.0016\end{array}$ & $\begin{array}{c}0.07488 \pm \\
0.00002\end{array}$ & TИMC $^{4}[17]$ \\
\hline GJ-1 & $\begin{array}{l}600.4 \pm 0.7^{(2)} \\
602.1 \pm 0.7^{(3)} \\
\end{array}$ & $\begin{array}{l}212- \\
422 \\
\end{array}$ & $19-37$ & $9-12$ & $\begin{array}{c}0.09761 \pm \\
0.00011 \\
\end{array}$ & $\begin{array}{c}0.8093 \pm \\
0.0009 \\
\end{array}$ & $\begin{array}{c}0.06014 \pm \\
0.00001 \\
\end{array}$ & TИМС [16] \\
\hline \multirow[t]{2}{*}{$\begin{array}{l}\text { Pleso- } \\
\text { vice }\end{array}$} & $337.13 \pm 0.37^{(2)}$ & $\begin{array}{l}465- \\
3084\end{array}$ & $44-523$ & $21-158$ & - & - & - & TИМС [13] \\
\hline & - & - & - & - & $\begin{array}{c}0.05372 \pm \\
0.00024\end{array}$ & $\begin{array}{c}0.3947 \pm \\
0.0022 \\
\end{array}$ & $\begin{array}{c}0.05319 \pm \\
0.00017 \\
\end{array}$ & BИМС $^{5}[20]$ \\
\hline
\end{tabular}

Примечание: (1) - возраст по конкордии; (2) - возраст, рассчитанный по изотопному отношению ${ }^{206} \mathrm{~Pb} /{ }^{238} U$ по уравнению радиоактивного распада; ${ }^{(3)}$ - возраст, рассчитанный по изотопному отношению ${ }^{207} \mathrm{~Pb} / 235 \mathrm{U}$ по уравнению радиоактивного распада; (4) - термоионизационная масс-спектрометрия; (4) - вторичная ионная масс-спектрометрия; «-»- нет данных. 
Таблица 2

Оптимизированные значения операционных параметров масс-спектрометра NexION $300 \mathrm{~S}$ и приставки для лазерной абляции NWR 213 (коллектор - детектор Фарадея)

Table 2

Optimized values of operating parameters of Neptune Plus mass spectrometer and NWR 213 attachment for laser ablation (Faraday detector is used as collector)

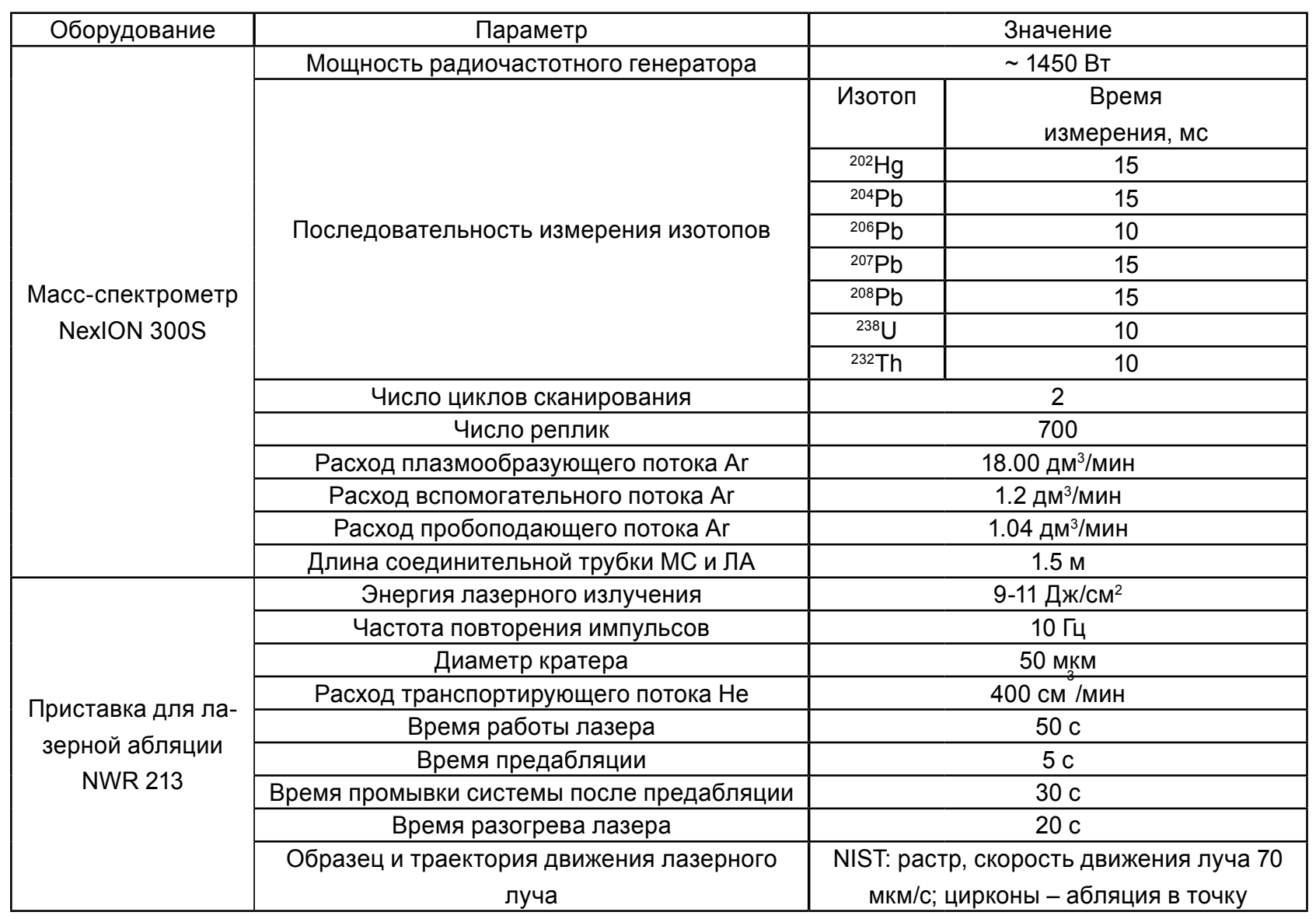

ректировку по внутреннему стандарту ТІ проводили, как и в работе [1], по линейному закону, используя соотношение ${ }^{205} \mathrm{TI} / 203 \mathrm{Tl}=2.3871 \pm 0.0013$ [22]. Значение сигнала изотопа ${ }^{235} \mathrm{U}$ рассчитывали из полученного значения сигнала изотопа ${ }^{238} \mathrm{U}$, используя естественную распространенность изотопов ${ }^{238} U / 235 U=137.7$.

Настройка операционных параметров квадрупольного МС (положение горелки; расход пробоподающего газа Ar; калибровка системы AutoLens для достижения максимальной эффективности пропускания интересующих изотопов) была произведена с помощью мультиэлементного раствора, содержащего 1 мкг/дм³ по Be, Ce, Fe, In, Li, Mg, Pb и U в 1 \% $\mathrm{HNO}_{3}$. В качестве параметров $\mathrm{MC}$, главным образом влияющих, по нашему мнению, на полученные значения сигналов изотопов при лазерной абляции, были выбраны следующие: время пребывания на массе и количество циклов сканирования. Оптимизацию данных параметров осуществляли, как и в работе [1], нахождением сочетания данных параметров при минимизации времени и погрешности измерения сигналов изотопов, а также при дости- жении максимума сигнала изотопов $\mathrm{Pb}, \mathrm{U}$ и Th на стандартном стекле NIST SRM 612.

\section{2. Приставка лазерной абляции}

Во многих публикациях [4-8, 21] наиболее часто используются следующие операционные параметры для лазерной приставки (при длине волны лазера 213 нм): частота 10 Гц; плотность энергии 4-11 Дж/см², в некоторых случаях до 25 Дж/см²; диаметр кратера от 20 до 40 мкм; скорость потока Не 0.3-0.6 дм³/мин.

Системы детектирования и временные параметры измерения многоколлекторного МС, использованного в [1], и квадрупольного МС, примененного в данной работе, значительно различаются между собой. В последнем случае это может сделать возможным проводить измерения при более низких значениях энергии, частоты и диаметра кратера ЛА, чем установленные в работе [1]. Поэтому в данной работе проведено повторное нахождение оптимальных параметров ЛА на примере цирконов Mud Tank [15] и Plesovice [13] (данные цирконы выбраны как наиболее отличающиеся по содержа- 
нию $\mathrm{U}$, Th и $\mathrm{Pb})$ : изучено влияние диаметра кратера абляции (25, 40 и 50 мкм), частоты повторения импульсов (5-20 Гц) и энергии лазерного излучения (5-40 Дж/см²) на уровень аналитического сигнала и величину эфффекта элементного фракционирования (см. раздел 3.2). Критерии выбора параметров ЛА приведены в [1]. Соответствие значений процентов от максимальной мощности энергии лазера и плотности энергии лазерного излучения в Дж/см² также приведено в работе [1]. Оптимизация перечисленных параметров (см. табл. 2) обеспечивает максимальную точность и минимальную неопределенность значений изотопных отношений.

Для удаления поверхностного загрязнения циркона применяли режим предабляции. Кроме того, использовали разогрев лазера перед началом абляции в течение 20 с, в это время не происходит взаимодействия лазерного излучения с поверхностью образца и регистрируется сигнал контрольного опыта (фоновый сигнал).

Измерения сигналов изотопов проводили методов «взятия в вилку» [23]. Реализовывали следующую последовательность измерения образцов: NIST, $91500_{1}$, MudTank ${ }_{1}$, GJ-1 1 , Plesovice ${ }_{1}$, NIST $_{2}$, 91500 ${ }_{2}$, MudTank ${ }_{2}$, GJ-1$_{2}$, Plesovice ${ }_{2}$ и т.д., ..., NIST $_{n}$. Сигнал контрольного образца (фоновый сигнал) измеряли для каждого кратера перед взаимодействием лазерного излучения с поверхностью образца.

\section{3. Результаты измерений}

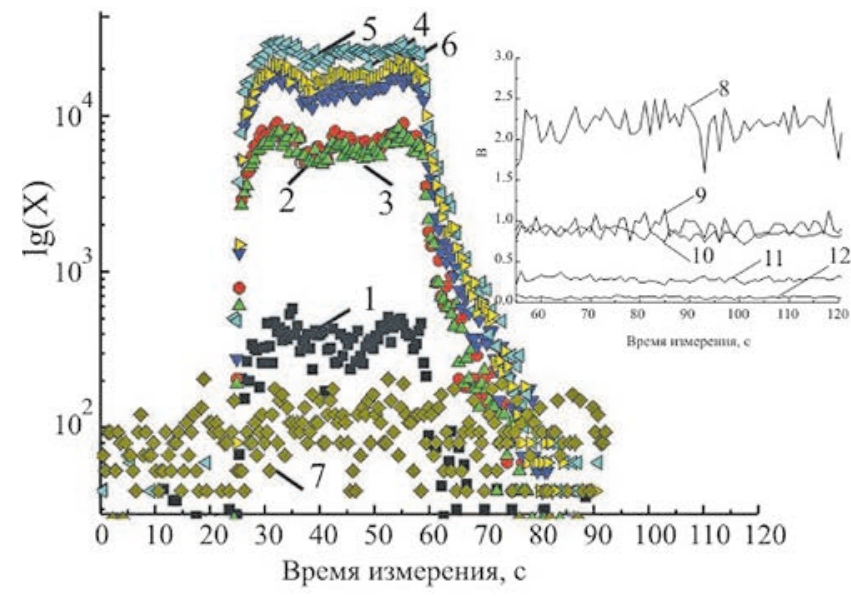

a

\section{1. Уровень сигнала холостого опыта, прецизионность и правильность определения изотопных отношений в стандарте NIST SRM 612}

Правильность разработанной схемы измерений, как и в случае [1], была проверена на примере определения изотопных отношений свинца в стандартном стекле NIST SRM 612 [14]. На рис. 1a представлены полученные с квадрупольным МС сигналы от изотопов $\mathrm{Hg}, \mathrm{Pb}, \mathrm{U}$ и Th для холостого опыта и стандарта NIST SRM 612. Аналогично работе [1], для холостого опыта фиксируется достаточно высокий уровень сигнала от изотопов $\mathrm{Hg}$ (вероятнее всего из инертных газов). Здесь и далее при сравнении результатов, полученных для сочетания квадрупольного MC NexION $300 S$ с приставкой ЛА NWR 213 и многоколлекторного MC Neptune Plus с приставкой ЛA NWR 213, использовали следующие сокращенные обозначения: NexION 3005 + NWR 213 и Neptune Plus + NWR 213 соответственно. Обсуждение результатов, полученных при сочетании Neptune Plus + NWR 213, проведено в [1].

Необходимо отметить, что неконтролируемые вариации сигналов во время измерения при сочетании NexION 300S + NWR 213 (рис. 1а) оказались значительно меньше, чем при сочетании Neptune Plus + NWR 213 (рис. 1б). По-видимому, это обусловлено значительно меньшим временем сбора сигналов изотопов квадрупольным МС при отсутствии

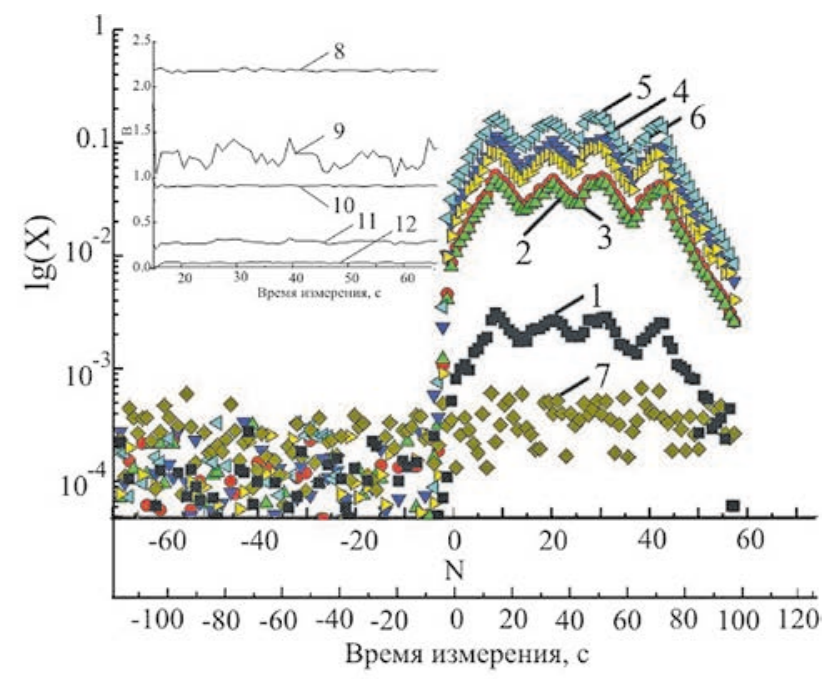

6

Рис. 1. Типичные сигналы изотопов (1) ${ }^{204}(\mathrm{~Pb}+\mathrm{Hg})$, (2) ${ }^{206} \mathrm{~Pb},(3){ }^{207} \mathrm{~Pb},(4){ }^{208} \mathrm{~Pb},(5){ }^{238} \mathrm{U}$, (6) ${ }^{232} \mathrm{Th},(7){ }^{202} \mathrm{Hg}$ и распространение изотопных отношений (8) ${ }^{208} \mathrm{~Pb} / 206 \mathrm{~Pb}$, (9) ${ }^{207} \mathrm{~Pb} /{ }^{206} \mathrm{~Pb}$, (10) ${ }^{208} \mathrm{~Pb} / 232 \mathrm{Th}$, (11) ${ }^{206} \mathrm{~Pb} /{ }^{238} \mathrm{U}$, (12) ${ }^{204} \mathrm{~Pb} / 206 \mathrm{~Pb}$ на стандартном стекле NIST 612, полученные при сочетании (а) NexION 300S + NWR 213 и (б) Neptune Plus + NWR 213, в зависимости от (а) времени измерения, (б) цикла измерения $N(-60 \div 0$ в контрольном опыте; $0 \div 60$ в стандарте) и времени измерения (-100 $\div 0$ в контрольном опыте; $0 \div 100$ в стандарте). $X$ - интенсивность сигнала изотопа в размерности: $\mathrm{a}-$ имп/c, 6 - В. Значение изотопного отношения - $B$

Fig. 1. Typical signals from isotopes (1) ${ }^{204}(\mathrm{~Pb}+\mathrm{Hg})$, (2) ${ }^{206} \mathrm{~Pb},(3){ }^{207} \mathrm{~Pb},(4){ }^{208} \mathrm{~Pb},(5){ }^{238} \mathrm{U},(6){ }^{232} \mathrm{Th},(7){ }^{202} \mathrm{Hg}$, and the estimated respective ratios $(8){ }^{208} \mathrm{~Pb} /{ }^{206} \mathrm{~Pb}$, (9) ${ }^{207} \mathrm{~Pb} / 206 \mathrm{~Pb}$, (10) ${ }^{208} \mathrm{~Pb} /{ }^{232} \mathrm{Th},(11){ }^{206} \mathrm{~Pb} / 238 \mathrm{U}$, (12) ${ }^{204} \mathrm{~Pb} / 206 \mathrm{~Pb}$ in standard NIST SRM 612 in combination of Neptune Plus + NWR 213 (a) and NexION 300S + NWR 213 (б) depending on (a) the time of measurement and (b) the cycle of $N(-60 \div 0$ for blank; $0 \div 60$ for standard) measurement and the time of measurement $(-100 \div 0$ for blank; $0 \div 100$ for standard). $X$ - the intensity of the isotope signal in (a) cps and (b) B. The value of the isotopic ratio $-B$ 
необходимости длительных затрат времени на переключение магнита между линиями измерения. Но, как показано на рис. 2a, это незначимо влияет на величины изотопных отношений, которые практически не изменяются в течение времени измерения (рис. 1а). Процедуры расчета изотопных отношений ${ }^{204} \mathrm{~Pb} /{ }^{206} \mathrm{~Pb},{ }^{207} \mathrm{~Pb} /{ }^{206} \mathrm{~Pb},{ }^{208} \mathrm{~Pb} /{ }^{06} \mathrm{~Pb}$, коррекции
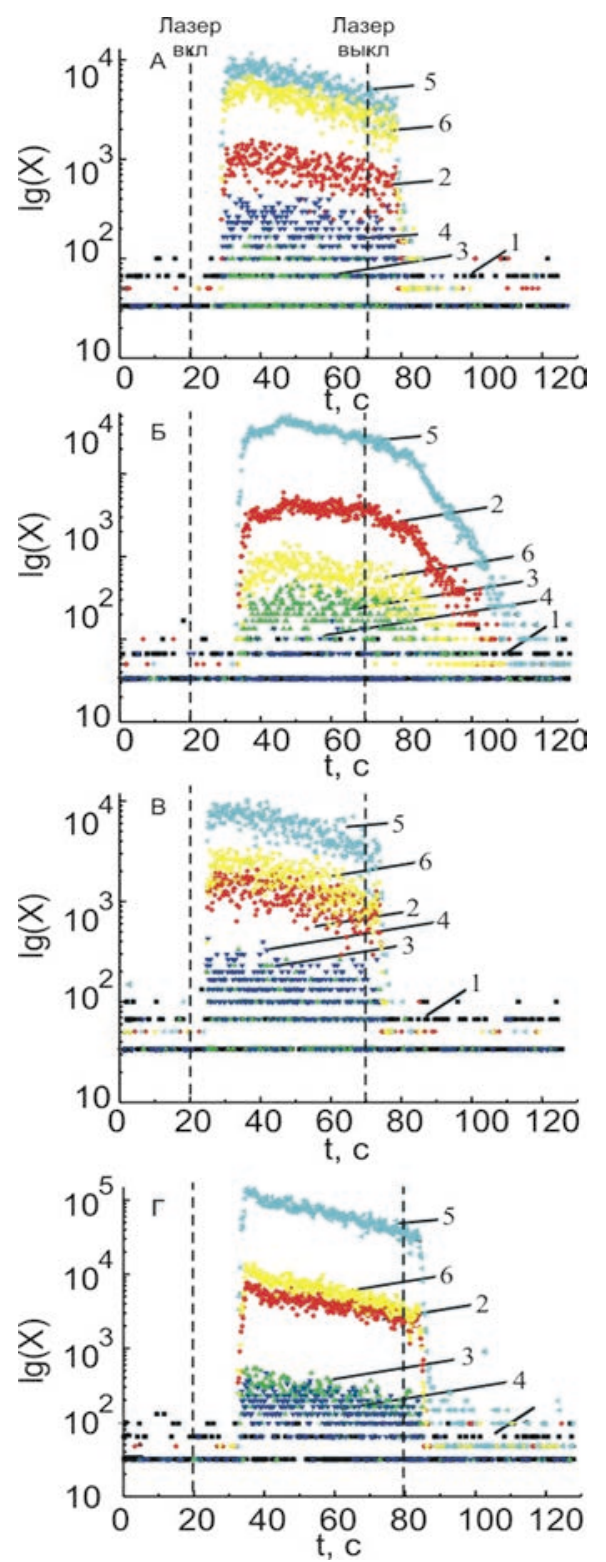

Рис. 2. Типичные сигналы от изотопов $\left({ }^{204} \mathrm{~Pb}+{ }^{204} \mathrm{Hg}\right)$ (1), ${ }^{206} \mathrm{~Pb}(2),{ }^{207} \mathrm{~Pb}(3),{ }^{208} \mathrm{~Pb}(4),{ }^{238} \mathrm{U}(5),{ }^{232} \mathrm{Th}(6)$ в цирконах Mud Tank (A), GJ-1 (Б), $91500(B)$ и Plesovice $(\Gamma)$ в зависимости от времени измерения $t$; $X-$ измеренное значение интенсивности сигнала изотопа, имп/с. Плотность энергии лазерного излучения - 9-11 Дж/ $\mathrm{cm}^{2}$; частота следования импульсов - 10 Гц; диаметр кратера - 50 мкм

Fig. 2. Typical signals from the isotopes $\left({ }^{204} \mathrm{~Pb}+{ }^{204} \mathrm{Hg}\right)(1)$, ${ }^{206} \mathrm{~Pb}(2),{ }^{207} \mathrm{~Pb}(3),{ }^{208} \mathrm{~Pb}(4),{ }^{238} \mathrm{U}(5),{ }^{232} \mathrm{Th}(6)$ in zircons Mud Tank $(A)$, GJ-1 (E), $91500(B)$ и Plesovice $(\Gamma)$ depending on the time of measurement $t ; X$ - the measured value of the isotope signal intensity, cps. Laser energy density 9-11 J/cm2; pulse repetition rate $-10 \mathrm{~Hz}$; crater diameter $-50 \mathrm{mkm}$ масс-дискриминации и статистической обработки результатов измерений описаны в [1].

Из рис. 1 и табл. 3 можно сделать вывод, что, как и прогнозировали, погрешность измерения для изотопных отношений $\mathrm{Pb} / \mathrm{Pb}$ при использовании сочетания NexION 300S + NWR 213 (последовательные измерения) выше, чем для Neptune Plus + NWR 213 (одновременные измерения [1]).

\section{2. Аналитические сигналы и изотопные отношения в природных стандартах цирконах; элементное фракционирование}

Эффректы элементного фракционирования компонентов пробы, искажающие результаты измерений, наблюдаются практически при любой реализации метода лазерной абляции, в том числе и при U-Pb датировании [1]. Решить данную проблему в значительной степени позволяет использование стандартного образца с близкими свойствами к исследуемому образцу [24].

Для оценки эффректа элементного фракционирования провели измерение имеющихся стандартных цирконов сочетанием NexION 300S + NWR 213 при операционных параметрах, представленных в табл. 2. На рис. 2 приведены полученные при этом типичные сигналы изотопов $\mathrm{Pb}, \mathrm{U}$ и Th в образцах природных цирконов. Во всех измеренных цирконах наблюдаются фолуктуации аналитических сигналов во времени, обусловленные элементным фракционированием. При этом форма сигнала существенно зависит от образца: для циркона Mud Tank cтационарное (закономерно и слабо меняющееся во времени) значение интенсивности (на примере сигналов изотопов ${ }^{238} \mathrm{U}$ и ${ }^{232} \mathrm{Th}$ ) устанавливается, как правило, в течение 15-17 с после начала абляции, для 91500 - в течение 5-7 с, а для GJ-1 и Plesovice - в течение 15-17 с. Время удаления испарившейся пробы из ячейки абляции после выключения лазера для рассматриваемых образцов циркона (на примере сигналов тех же изотопов) различно и составляет для Mud Tank - 10-12 с, для 91500 - 7-10 с, для GJ-1 - 40-42 с и для Plesovice - 17-19 с. Стоит отметить, что на время установления стационарного сигнала и удаления испаренной пробы из ячейки, кроме свойств образца, также влияет длина соединительной трубки МС и приставки для ЛА (в нашем случае - 1.5 м, см. табл. 2).

Для определения оптимальных параметров работы приставки для ЛА на примере цирконов Mud Tank [15] и Plesovice [13], характеризующихся низким и высоким содержанием $\mathrm{U}$, Th и $\mathrm{Pb}$ соответственно (см. табл. 1), изучено влияние диаметра кратера абляции (25, 40 и 50 мкм), частоты повторения импульсов (5-20 Гц) и энергии лазерного излучения (5-40 Дж/см²) на уровень аналитического сигнала и величину эффекта элементного фракционирования. Для количественной характеристики эффекта элементного фракционирования, вновь 
Сопоставление значений изотопных отношений свинца, стандартных отклонений результатов измерения $(s, \%)$ и показателя правильности измерения ( $\pm \Delta c, \%)$ в стандартном стекле NISTSRM 612 для сочетаний Neptune Plus + NWR 213 и NexION 300S + NWR 213. $n$ - число измерений

Table 3

Values of the lead isotope ratios in standard glass NIST SRM 612, the standard deviation of the measurement results $(s, \%)$ and the precision of the measurement $( \pm \Delta c, \%)$ in combination of Neptune Plus + NWR 213 and NexION 300S + NWR 213. $n$ - number of measurements

\begin{tabular}{|c|c|c|c|c|c|c|c|}
\hline \multirow{2}{*}{$\begin{array}{c}\text { Изотопное от- } \\
\text { ношение }\end{array}$} & \multirow{2}{*}{$\begin{array}{c}\text { Опорное зна- } \\
\text { чение [14] }\end{array}$} & \multicolumn{3}{|c|}{ Neptune Plus + NWR 213, $n=12$} & \multicolumn{3}{|c|}{ NexION 300S + NWR 213, $n=10$} \\
\hline & & $\begin{array}{c}\text { Измеренное значе- } \\
\text { ние }( \pm 2 \sigma)\end{array}$ & $s, \%$ & $\pm \Delta_{c}, \%$ & $\begin{array}{c}\text { Измеренное значе- } \\
\text { ние }( \pm 1 \sigma)\end{array}$ & $s, \%$ & $\pm \Delta_{c}, \%$ \\
\hline${ }^{204} \mathrm{~Pb} / 206 \mathrm{~Pb}$ & 0.059 & $0.0574 \pm 0.0009$ & 5 & 3 & $0.0610 \pm 0.0009$ & 6.2 & 3.3 \\
\hline${ }^{207} \mathrm{~Pb} / 206 \mathrm{~Pb}$ & 0.9073 & $0.890 \pm 0.002$ & 0.4 & 2 & $0.912 \pm 0.042$ & 1.4 & 0.5 \\
\hline${ }^{208} \mathrm{~Pb} / 206 \mathrm{~Pb}$ & 2.1647 & $2.141 \pm 0.009$ & 0.5 & 0.6 & $2.158 \pm 0.009$ & 1.7 & 0.3 \\
\hline${ }^{207} \mathrm{~Pb} /{ }^{204} \mathrm{~Pb}$ & 15.511 & $15.2 \pm 0.2$ & 4 & 2 & $15.0 \pm 0.2$ & 4.7 & 3.3 \\
\hline${ }^{208} \mathrm{~Pb} /{ }^{204} \mathrm{~Pb}$ & 37.005 & $36.6 \pm 0.6$ & 4 & 1 & $35.5 \pm 0.5$ & 6.3 & 4.3 \\
\hline
\end{tabular}

использовали, как и в [1], параметр п, рассчитанный как тангенс угла наклона линии регрессии (зависимость значения изотопного отношения от времени измерения), нормированный на значение точки ее пересечения с осью ординат [25]. Значение п характеризует относительное изменение изотопного отношения во время абляции, что позволяет проводить сравнение величины п для разных образцов циркона.

На рис. 3 для цирконов Mud Tank и Plesovice представлены зависимости параметра п изотопного отношения ${ }^{206} \mathrm{~Pb} /{ }^{238} \mathrm{U}$ от плотности энергии лазерного излучения (рис. За, при частоте повто-

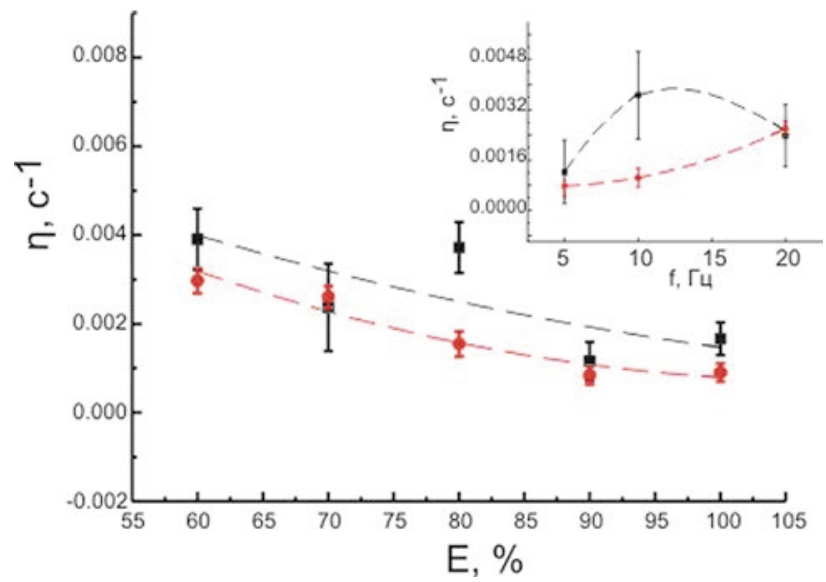

Рис. 3. Величина параметра элементного фракционирования $\left(\eta, \mathrm{c}^{-1}\right)$ для измеренного изотопного отношения ${ }^{206} \mathrm{~Pb} /{ }^{238} U$ в цирконе Mud Tank (A, B) и Plesovice

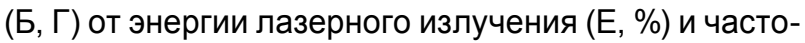
ты повторения импульсов. Пунктирные линии - для наглядности проявления тенденции. Погрешность 1 б. Диаметр кратера 50 мкм

Fig. 3. The value of elemental fractionation $\left(\eta, s^{-1}\right)$ for the measured ${ }^{206} \mathrm{~Pb} /{ }^{238} \mathrm{U}$ isotopic ratio in zircon Mud Tank (A, $B)$ and Plesovice $(Б, \Gamma)$, and the energy of laser ablation $(E, \%)$ and the pulse repetition rate. Dashed lines show the illustration of the trend. The error is $1 \sigma$. The crater diameter is $50 \mathrm{mkm}$ рения импульсов 20 Гц) и от частоты повторения импульсов (рис. 3б, при энергии лазера 70 \%); диаметр кратера составлял 50 мкм. Видно, что параметр п снижается с ростом плотности энергии и с уменьшением частоты следования импульсов. Однако при ультранизких значениях частоты следования импульсов (4 и 5 Гц) и плотности энергии (около 4 Дж/см²) увеличивается относительная погрешность измерения изотопных отношений, а при чрезмерно высоких значениях указанных параметров (21 Дж/см², 20 Гц) снижается качество кратера (на его стенках появляются неровности, следы разбрызгивания, бортики).

Аналогичные выводы были сделаны в [1]. Поэтому для достижения наилучших результатов целесообразен выбор некоторых средних значений. Отметим, что снижение параметра элементного фракционирования может быть также достигнуто в ряде случаев путем увеличения диаметра кратеpa; так, для образцов GJ-1 и Plesovice имеет место снижение параметра п примерно в два раза при переходе от кратеров диаметром 25 мкм к кратерам 50 мкм (табл. 4).

Для оценки влияния структурного состояния образца на характеристики элементного фракционирования мы сопоставили значения параметра п, полученного при операционных параметрах $80 \%$, 10 Гц, 25 мкм и $80 \%, 10$ Гц, 50 мкм в рамках одной измерительной сессии, для изотопного отношения ${ }^{206} \mathrm{~Pb} /{ }^{238} U$ в природных цирконах со степенью их радиационной деструкции за счет альфа-распада радиоактивных примесей урана и тория (табл. 4). Последнюю оценивали по значению накопленной радиационной альфа-дозы (расчет по содержанию $U$, Th и возрасту цирконов в соответствии c [26]), а также, используя подход [27], по усредненным по пяти точкам поверхности экспериментальным значениям ширины на половине высоты (FWHM) пика $1008 \mathrm{~cm}^{-1}$ асимметричных валентных колебаний $\mathrm{v}_{3}\left(\mathrm{SiO}_{4}\right), \mathrm{B}_{1 \mathrm{~g}}$ в рамановских спектрах. Coгласно этим оценкам, степень радиационного по- 
Таблица 4

Параметры элементного фракционирования изотопного отношения ${ }^{206} \mathrm{~Pb} / 238 \mathrm{U}$ при лазерной абляции цирконов с различным диаметром кратера $d$ (энергия лазерного излучения - $80 \%$, частота повторения импульсов - 10 Гц) и характеристики их авторадиационного повреждения - расчетные значения накопленной радиационной дозы облучения ( $X$, а-распадов/г) и экспериментальные значения ширины (FWHM) рамановской линии $1008 \mathrm{~cm}^{-1}$

Table 4

Characteristics of elemental fractionation of isotope ratio ${ }^{206} \mathrm{~Pb} / 238 \mathrm{U}$ during the laser ablation of zircons with different crater diameter $d$ (energy $-80 \%$, pulse repetition rate $-10 \mathrm{~Hz}$ ) and the characteristics of their autoradiation damage - the calculated values of the accumulated radiation dose and the experimental values of the width (FWHM) of Raman line $1008 \mathrm{~cm}^{-1}$

\begin{tabular}{|c|c|c|c|c|c|c|c|}
\hline \multirow[t]{2}{*}{ № } & \multirow[t]{2}{*}{ Циркон } & \multicolumn{2}{|c|}{$\begin{array}{c}\text { Изотопное отношение, экс- } \\
\text { траполированное к моменту } \\
\text { начала абляции }{ }^{(1)}\end{array}$} & \multicolumn{2}{|c|}{$\begin{array}{c}\text { Параметр элементного фракцио- } \\
\text { нирования } \eta^{(2)}\end{array}$} & \multirow[t]{2}{*}{$\begin{array}{l}X / 10^{18} \\
(3)\end{array}$} & \multirow[t]{2}{*}{$\begin{array}{l}\text { FWHM, } \\
\mathrm{CM}^{-1}(4)\end{array}$} \\
\hline & & $d=25$ & $d=50$ & $d=25$ & $d=50$ & & \\
\hline 1 & Mud Tank & $0.130 \pm 0.003$ & $0.138 \pm 0.002$ & $0.0002 \pm 0.0003$ & $0.0003 \pm 0.0001$ & $0.02-0.12$ & 2.1 \\
\hline 2 & 91500 & $0.203 \pm 0.003$ & $0.206 \pm 0.002$ & $0.0002 \pm 0.0002$ & $0.00033 \pm 0.00006$ & $0.31-0.45$ & $\begin{array}{c}3.8 \\
2.7-7.3^{(5)}\end{array}$ \\
\hline 3 & GJ-1 & $0.104 \pm 0.002$ & $0.1222 \pm 0.0009$ & $0.0006 \pm 0.0002$ & $0.00023 \pm 0.00004$ & $0.56-1.12$ & 6.7 \\
\hline 4 & Plesovice & $0.049 \pm 0.004$ & $0.060 \pm 0.002$ & $0.0008 \pm 0.0005$ & $0.00038 \pm 0.00009$ & $1.24-8.23$ & $\begin{array}{c}11.0 \\
7-30^{(6)}\end{array}$ \\
\hline
\end{tabular}

Примечание: (1) - усредненное по пяти единичным измерениям значение точки пересечения с осью ординат (погрешность 1б) линии регрессии изотопных отношений от времени в процессе абляции; (2) - усредненная по пяти единичным измерениям величина тангенса наклона линии регрессии, нормированная на значение точки ее пересечения с осью ординат (погрешность 1б); (3) - значения накопленной дозы, рассчитанные по содержанию U, Th и возрасту цирконов (см. табл. 1) в соответствии с [26]; (4) - средние (по 5 точкам поверхности образцов) экспериментальные значения ширины на половине высоты (FWHM) пика 1008 см$^{-1}$ асимметричных валентных колебаний $\mathrm{v}_{3}\left(\mathrm{SiO}_{4}\right), \mathrm{B}_{1 \mathrm{~g}}$ в рамановских спектрах исследованных цирконов; ${ }^{(5)}$ - FWHM по данным [18]; (6) - FWHM по данным [13].

Таблица 5

Значение возраста стандартных цирконов при оптимизированных значениях операционных параметров по изотопным отношениям ${ }^{206} \mathrm{~Pb} /{ }^{238} \mathrm{U} u^{207} \mathrm{~Pb} / 235 \mathrm{U}$ и по конкордии ( $n$ - количество измерений, СКВО - среднее квадратичное взвешенное отклонение) для сочетания Neptune Plus + NWR 213 и для сочетания NexION $300 S+N W R 213$

Table 5

The age of standard zircons at optimized operating parameters, calculated by the developed algorithm of isotope ratios, by ${ }^{206} \mathrm{~Pb} /{ }^{238} \mathrm{U}$ and ${ }^{207} \mathrm{~Pb} /{ }^{235} \mathrm{U}$ isotope ratios and by Concordia ( $n$ = number of measurements, MSWD - middle square weighted deviations) in combination of Neptune Plus + NWR 213 and NexION 300S + NWR 213

\begin{tabular}{|c|c|c|c|c|c|c|}
\hline \multirow{2}{*}{$\begin{array}{c}\text { MC + пристав- } \\
\text { ка ЛА }\end{array}$} & \multirow{2}{*}{ Образец } & \multirow{2}{*}{ Стандарт } & \multirow{2}{*}{$n$} & \multicolumn{3}{|c|}{ Значение возраста, млн. лет ( $\pm 2 \sigma, \%)$} \\
\hline & & & & ${ }^{206} \mathrm{~Pb} /{ }^{238} \mathrm{U}$ & ${ }^{207} \mathrm{~Pb} /{ }^{235} \mathrm{U}$ & Конкордия \\
\hline \multirow{3}{*}{$\begin{array}{l}\text { Neptune Plus + } \\
\text { NWR } 213\end{array}$} & Mud Tank & 91500 & 7 & $\begin{array}{c}737 \pm 14 \\
(2 \%)\end{array}$ & $\begin{array}{c}737 \pm 42 \\
(6 \%)\end{array}$ & $\begin{array}{c}735 \pm 12 \\
(\text { СКВО }=1.2,2 \%)\end{array}$ \\
\hline & 91500 & Mud Tank & 7 & $\begin{array}{c}1052 \pm 20 \\
(2 \%) \\
\end{array}$ & $\begin{array}{c}1053 \pm 60 \\
(6 \%) \\
\end{array}$ & $\begin{array}{c}1054 \pm 17 \\
(\text { СКВО }=1.05,2 \%)\end{array}$ \\
\hline & GJ-1 & 91500 & 7 & $\begin{array}{c}599 \pm 23 \\
(4 \%)\end{array}$ & $\begin{array}{c}543 \pm 47 \\
(9 \%) \\
\end{array}$ & $\begin{array}{c}606 \pm 31 \\
(\text { СКВО }=0.38,5 \%) \\
\end{array}$ \\
\hline \multirow{4}{*}{$\begin{array}{c}\text { NexION } 300 S+ \\
\text { NWR } 213\end{array}$} & Mud Tank & GJ-1 & 5 & $\begin{array}{c}727 \pm 20 \\
(3 \%)\end{array}$ & $\begin{array}{c}677 \pm 56 \\
(8 \%)\end{array}$ & $\begin{array}{c}719 \pm 17 \\
(\text { СКВО }=0.91,2.4 \%)\end{array}$ \\
\hline & 91500 & GJ-1 & 9 & $\begin{array}{c}1058 \pm 17 \\
(2 \%)\end{array}$ & $\begin{array}{c}1100 \pm 51 \\
(5 \%)\end{array}$ & $\begin{array}{c}1065 \pm 14 \\
(\mathrm{CKBO}=1.19,1.3 \%)\end{array}$ \\
\hline & GJ-1 & - & 7 & $\begin{array}{c}601 \pm 10 \\
(2 \%)\end{array}$ & $\begin{array}{c}602 \pm 27 \\
(5 \%) \\
\end{array}$ & $\begin{array}{c}600.8 \pm 8.8 \\
(\mathrm{CKBO}=0.017,1.5 \%)\end{array}$ \\
\hline & Plesovice & GJ-1 & 5 & $\begin{array}{c}337.5 \pm 8.4 \\
(3 \%)\end{array}$ & $\begin{array}{c}330 \pm 25 \\
(7 \%)\end{array}$ & $\begin{array}{c}336.6 \pm 7.7 \\
(\mathrm{CKBO}=0.24,2.3 \%)\end{array}$ \\
\hline
\end{tabular}


вреждения стандартных образцов увеличивается в ряду Mud Tank < $91500<$ GJ < Plesovice.

При измерениях изотопных отношений с диаметром кратера 25 мкм значение параметра $\eta$ увеличивается при переходе от цирконов Mud Tank и 91500, имеющих низкую степень радиационного повреждения, к цирконам GJ и Plesovice, обладающих средней и высокой степенью повреждения. Такая зависимость соответствует представлениям [25] об увеличении эфффективности испарения и элементного фракционирования с ростом степени авторадиационного повреждения цирконов. Однако при измерениях с диаметром кратера 50 мкм параметр элементного фракционирования для отношения ${ }^{206} \mathrm{~Pb} /{ }^{238} U$ всех цирконов становится практически одинаковым. Этот фракт позволяет предположить, что при работе с большими кратерами и объемами испаренной пробы значимость влияния структурного состояния образца на эфрфекты элементного фракционирования снижается.

С учетом полученных данных о влиянии операционных параметров сочетания NexION $3005+$ NWR 213 на значения ๆ, а также погрешности измерения изотопных отношений и внешний вид кратеров абляции, оптимальными едиными параметрами для измерения изотопных отношений $\mathrm{Pb} / \mathrm{U}$ и $\mathrm{Pb} / \mathrm{Th}$ во всех изученных цирконах выбраны следующие: плотность энергии лазерного излучения 9-11 Дж/см ${ }^{2}$ (или $60 \%$ от максимальной мощности), частота повторений импульсов 10 Гц, диаметр кратера 50 мкм. Это расширяет возможности разработанной методики определения возраста цирконов по сравнению c [1] (Neptune Plus + NWR 213), где, для получения стабильного сигнала и уменьшения погрешности измерения изотопных отношений циркона Mud Tank c низким содержанием $\mathrm{Pb}, \mathrm{U}$ и Th, необходимо было использовать индивидуальные условия ЛА.

\section{3 Корректировка эффректов дискриминации ионов по массе, дрейфа прибора и оценка содержания нерадиогенного свинца}

Ранее [1] нами была разработана схема расчета изотопных отношений $\mathrm{Pb} / \mathrm{U}$ в цирконах c последующим определением возраста, основным недостатком которой было длительное время обработки данных. В данной работе для обработки полученных данных была использована программа GLITTER (демо-версия 4.4.4) и схема расчета изотопных отношений выглядит следующим образом:

Расчет изотопных отношений $\mathrm{Pb} / \mathrm{Pb}, \mathrm{Pb} / \mathrm{U}$ и $\mathrm{Pb} / \mathrm{Th}$ с помощью программы GLITTER (демо-версия 4.4.4), который включает процедуры:

- выбор стандартного образца;

- проверка на фракционирование (осуществляется автоматически при загрузке данных в программу, производится проверка фракционирования для изотопов одного элемента, которое должно составлять не более $5 \%$ );
- выбор типа интерполяции результатов стандартного образца для проведения корректировки инструментального дрейфа (в данной работе использовали функцию Linear fit);

- выбор интервалов для расчета фонового и полезного сигналов;

- проверка на наличие выбросов.

Корректировка на присутствие нерадиогенного свинца в программе ComPbCorr\#3_18 [28];

Построение конкордии в программе Excel c встроенным пакетом Isoplot (версия 4.15).

\section{4. Расчет возраста стандартных образцов природного циркона}

Расчет возраста цирконов был проведен по скорректированным значениям отношений ${ }^{206} \mathrm{~Pb} / 238 \mathrm{U}$ $n^{207} \mathrm{~Pb} / 235 \mathrm{U}$ по уравнениям радиационного распада (см. табл. 5). На рис. 4 сопоставлены значения стан-
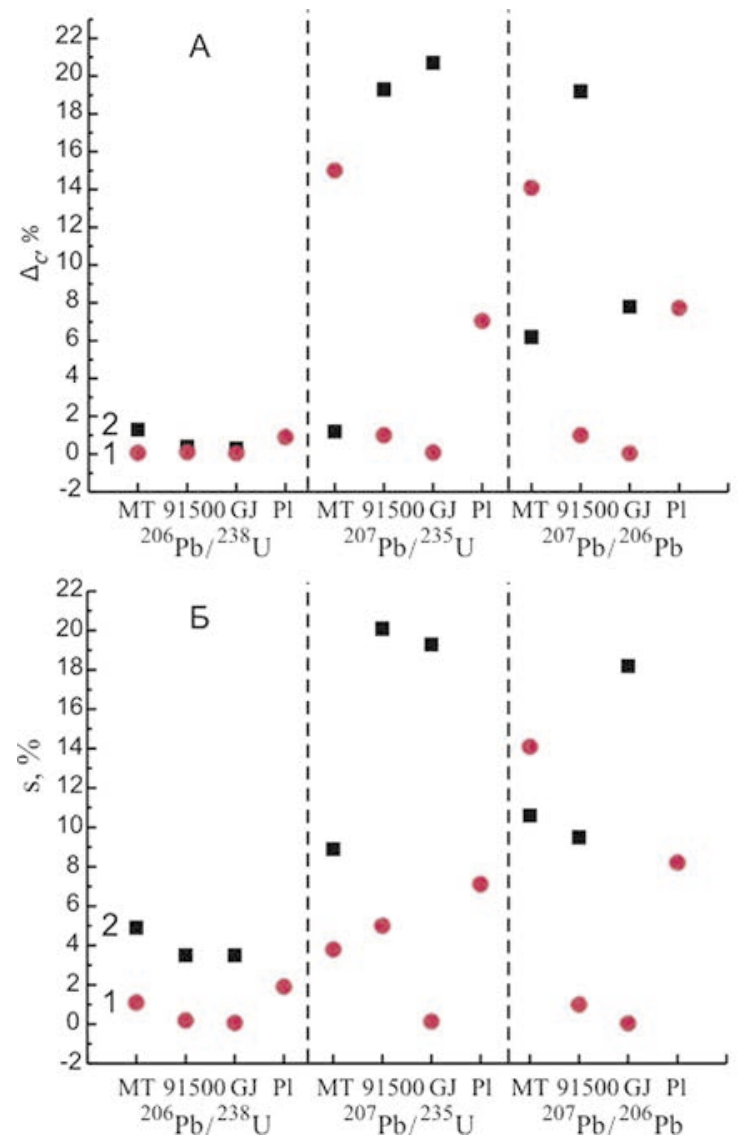

Рис. 4. Значения показателя правильности измерения $\left( \pm \Delta_{c}, \%, A\right)$ и стандартного отклонения (повторяемость) результатов измерения $(s, \%$, Б) для изотопных отношений $\mathrm{Pb} / \mathrm{U}$ и $\mathrm{Pb} / \mathrm{Pb}$ в стандартных цирконах при сочетании (1) NexION 300S + NWR 213 и (2) Neptune Plus + NWR 213. Количество кратеров - 3. MT - Mud Tank, PI - Plesovice

Fig. 4. The precision of measurement results $\left( \pm \Delta_{c}, \%, A\right)$, the relative standard deviation (repeatability) of measurement $(s, \%$, Б) for the measured $\mathrm{Pb} / \mathrm{U}$ и $\mathrm{Pb} / \mathrm{Pb}$ isotopic ratios in standard zircon in combination of Neptune Plus + NWR 213 and NexION 300S + NWR 213. The number of craters -3. MT - Mud Tank, PI - Plesovice 
дартного отклонения результатов измерения (s, \%) и показателя правильности измерения $\left( \pm \Delta_{c}, \%\right)$ для изотопных отношений $\mathrm{Pb} / \mathrm{U}$ и $\mathrm{Pb} / \mathrm{Pb}$ в стандартных образцах циркона при оптимизированных значениях МС и приставки для ЛА при сочетании Neptune Plus + NWR 213 и NexION 300S + NWR 213. Результаты для каждого сочетания приборов получены в рамках одной измерительной сессии. На рис. 5а-г представлена диаграмма ${ }^{206} \mathrm{~Pb} / 238 \mathrm{U}$ от ${ }^{207} \mathrm{~Pb} /{ }^{235} \mathrm{U}$ для цирконов Mud Tank, 91500 и GJ-1. В качестве стандартного образца использовали циркон GJ-1. Полученные нами значения возраста цирконов согласуются с литературными данными (см. табл. 1).

Анализ данных, представленных в табл. 5 и на рис. 4, приводит к выводу, что для U-Pb датирования цирконов использование сочетания NexION $300 S$ + NWR 213 позволяет снизить погрешность измерения изотопных отношений и улучшить зна- чение показателя правильности измерения изотопных отношений по сравнению с использованным ранее [1] сочетанием Neptune Plus + NWR 213. Таким образом, для дальнейших исследований цирконов методом ЛА-ИСП-МС нами выбран масс-спектрометр NexION 300S (PerkinElmer) и приставка для лазерной абляции NWR 213 (ESI).

\section{5 Датировка природного циркона из гранитоидов}

Представленный в работе алгоритм измерения (NexION 300S + NWR 213) и расчета изотопных отношений был опробован на микрокристаллах акцессорного циркона пробы К2074 из коллекции А.А. Краснобаева, выделенных из гранитоидов Новоусмановского массива (150 м к СВ $14^{\circ}$ от вершины г. Артлыш с отметкой 605 м). Детальное минералого-геохимическое исследование
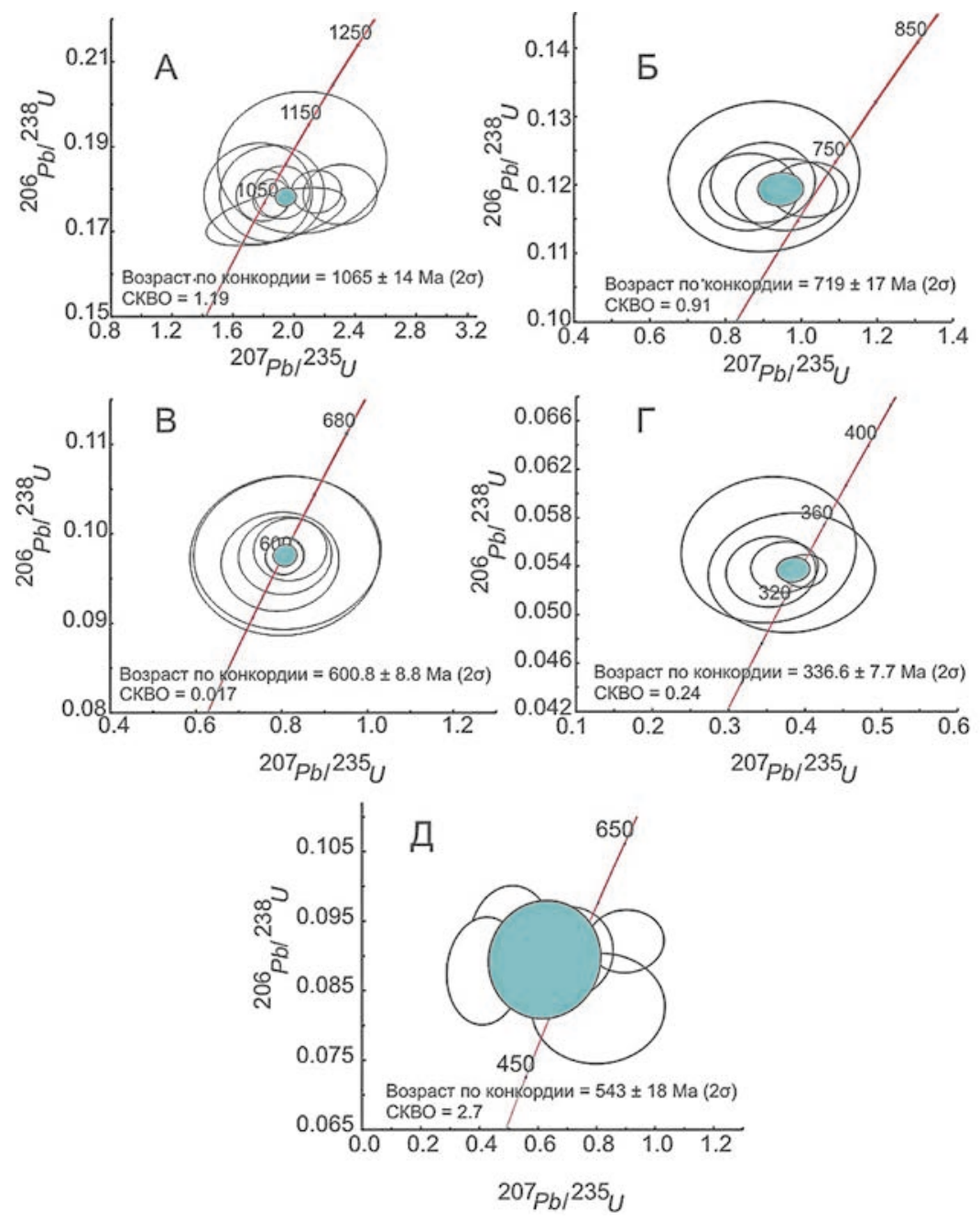

Рис. 5. Диаграмма ${ }^{206} \mathrm{~Pb} / 238 U$ vs ${ }^{207} \mathrm{~Pb} / 235 U$ для стандартных образцов циркона: a - 91500; б - Mud Tank; в - GJ-1; г - Plesovice; д - циркон пробы K2074. Стандартный образец - GJ-1. Незалитый эллипс - значения изотопных отношений, соответствующие единичному измерению кратера; синий эллипс - средневзвешенные значения; красная линия - конкордия; погрешность - 2б. СКВО - среднеквадратичное взвешенное отклонение результатов измерения

Fig. 5. ${ }^{206} \mathrm{~Pb} /{ }^{238} U$ vs ${ }^{207} \mathrm{~Pb} /{ }^{235} U$ diagram for zircon standards $91500(A)$, Mud Tank $(Б)$, GJ-1 $(B)$, Plesovice $(\Gamma)$ and zircon of sample K2017 (Д). Zircon standard - GJ-1.Unfilled ellipse designates the isotope ratio values corresponding to the single measurement of the crater; blue ellipse is the weighted average; red line represents Concordia; error is $2 \sigma$. MSWD - middle square weighted deviations of the measurement result 

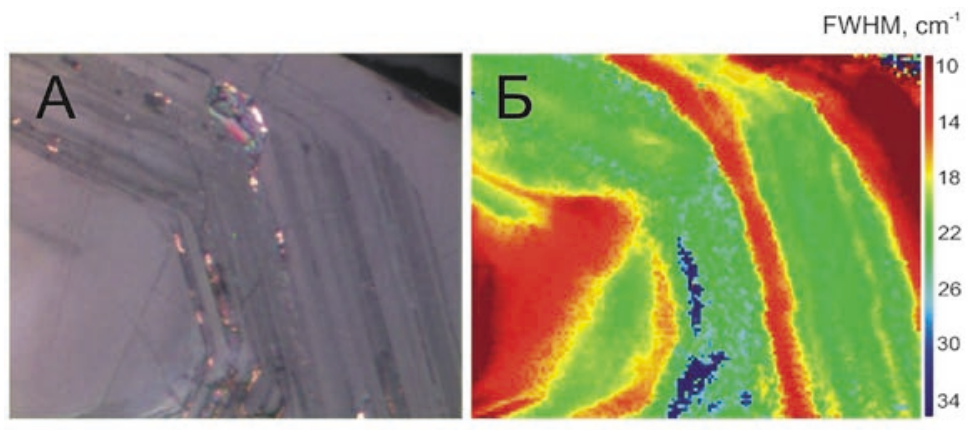

$50 \mu \mathrm{m}$

Рис. 6. Оптическое изображение в отраженном свете фрагмента типичного зерна циркона пробы К2074 из гранитоидов Новоусмановского массива (A) и результат картирования этого фрагмента методом рамановской спектроскопии по ширине линии $1008 \mathrm{~cm}^{-1}($ Б), отражающей степень радиационного повреждения структуры циркона Fig. 6. Optical image in the reflected light of a typical fragment of zircon grains samples K2074 from the granitoids of Novousmanovskii massif $(A)$, and the result of mapping this fragment by Raman spectroscopy on the line width $1008 \mathrm{~cm}^{-1}$ (B), which reflects the degree of radiation damage to the structure of zircon

циркона, состав примесных РЗЭ, а также данные его изотопного U-Pb-датирования представлены в работе [29]. Измерения методом рамановской спектроскопии показали, что исследуемые зерна неоднородны по степени радиационного повреждения, которая изменяется от средней до высокой в пределах одного зерна; ширина рамановской линии $1008 \mathrm{~cm}^{-1}$ варьирует от 10 до 34 $\mathrm{CM}^{-1}$; при работе с кратерами диаметром 50 мкм абляция вещества происходит одновременно из зон различной степени повреждения (см. рис. 6). Зерна характеризуются широкими вариациями степени радиационного повреждения структуры: от средней степени во внутренней части кристалла (красный цвет, левый нижний край) и в тонкой внешней оболочке (красный цвет, правый верхний край) до высокой степени в широкой зональной кайме, отделяющей внутреннюю часть зерна от внешней оболочки (желтый, зеленый, синий цвета).

В качестве стандартного образца при изотопных измерениях использован образец средней степени радиационной деструкции GJ-1. Полученное нами значение U-Pb возраста по конкордии (рис. 5д) составило $543 \pm 18$ млн. лет (СКВО = 2.7, $2 \sigma=3.3 \%)$, что удовлетворительно согласуется с изотопными данными $542.9 \pm 6.6$ млн. лет (СКВО =0.0042, $2 \sigma=1.2 \%$, масс-спектрометр SHRIMP).

\section{Выводы}

В работе представлены этапы разработки ЛА-ИСП-МС-методики анализа изотопного состава $\mathrm{Pb}$ и U в цирконах и процедура обработки данных, реализованные в Центре коллективного пользования УрО РАН «Геоаналитик» при ИГГУрО PAH на квадрупольном ИСП-MC NexION $300 \mathrm{~S}$ с приставкой для ЛА NWR 213. Определены оптимальные параметры MC (расходы плазмообразующего, вспомогательного и пробоподающего потоков газа $\mathrm{Ar}$, мощность радиочастотного гене- ратора, время пребывания на массе, время полного анализа единичного кратера) и приставки для ЛА (поток транспортирующего газа Не, плотность энергии лазерного излучения, частота повторения импульсов, диаметр кратера) для измерения изотопных отношений $\mathrm{Pb} / \mathrm{U}, \mathrm{Pb} / \mathrm{Th}$ и $\mathrm{Pb} / \mathrm{Pb}$ в цирконе. Выполнен анализ неопределенности измерения изотопных отношений. Представлена сравнительная характеристика использования МС и приставки для ЛА при сочетании Neptune Plus + NWR 213 и NexION $300 S$ + NWR 213 применительно к методике U-Pb датирования цирконов. Отработана схема определения изотопных отношений $\mathrm{Pb} / \mathrm{U}, \mathrm{Pb} / \mathrm{Th}$ и $\mathrm{Pb} / \mathrm{Pb}$ с последующим расчетом их возраста. Показаны метрологические характеристики разработанной методики анализа возраста цирконов. Предложенная схема использована при датировании стандартных образцов циркона 91500, Mud Tank, GJ-1 и Plesovice: значение их возраста по конкордии составило $1065 \pm 14$ млн. лет (СКВО = 1.19, 2б=1.3\%), $719 \pm 17$ млн. лет (СКВО $=0.91,2 \sigma=2.4 \%), 600.8 \pm 8.8 \mathrm{млн.} \mathrm{лет} \mathrm{(СКВО} \mathrm{=}$ $0.017,2 \sigma=1.5 \%)$ и $336.6 \pm 7.7 \mathrm{млн.} \mathrm{лет} \mathrm{(СКВО} \mathrm{=}$ $0.24,2 \sigma=2.3 \%$ ), соответственно, что согласуется в пределах неопределенности со значениями, полученными в других лабораториях. Процедуры измерения и расчета изотопных отношений, представленные в работе, опробованы при датировке акцессорного циркона из гранитоидов Новоусмановского массива (Урал); получено значение U-Pb возраста по конкордии $543 \pm 18$ млн. лет (СКВО = $2.7,2 \sigma=3.3 \%)$, что удовлетворительно согласуется с данными SHRIMP.

\section{Благодарности}

Авторы признательны А.А. Краснобаеву за предоставленные пробы цирконов и полезное обсуждение.

Работа выполнена при поддержке гранта РНФ № 16-17-10283, гранта РФФИ №14-05-00172 
в Центре коллективного пользования УрО РАН «Геоаналитик»

\section{Acknowledgement}

The authors are grateful to A.A. Krasnobaev for providing the zircon samples and useful discussion.

The study was performed as part of RSF grant № 16-17-10283, RFBR grant №14-05-00172 at the «Geoanalitik» Analytical Center of UB RAS.

\section{ЛИТЕРАТУРА}

1. Зайцева М.В., Пупышев А.А., Щапова Ю.В., Вотяков С.Л. Методические аспекты U/Pb датирования цирконов на многоколлекторном масс-спектрометре с индуктивно-связанной плазмой NeptunePlus с приставкой для лазерной абляции NWR 213 // Аналитика и контроль. 2016. T. 10, № 2. C. 121-137

2. Longerich $\mathrm{H}$. Laser ablation-inductively coupled plasmamass spectrometry (LA-ICP-MS); An introduction // Laser Ablation-ICP-MS in the Earth Sciences / Ed. by Sylvester P. Mineralogical Association of Canada. 2008. V. 40. P. 1-18. 3. Vanhaecke F., Kyser K. The Isotopic Composition of the Elements // Isotopic Analysis. Fundamentals and Applications Using ICP-MS / Ed. by Vanhaecke F., Degryse P.WILEY-VCH Verlag GmbH \& Co. KGaA, Weinheim.2012. P. 1-29

4. Determination of $\mathrm{U}-\mathrm{Pb}$ age and rare earth element concentrations of zircons from Cenozoic intrusions in northeastern China by laser ablation ICP-MS / Y. Honglin [et. al.] // Chinese Science Bulletin. 2003. V. 48, № 22. P. 2411-2421.

5. Rapid and simultaneous determination of multi-element abundances and $\mathrm{U}-\mathrm{Pb}$ age for zircon crystal using UV laser ablation ICP-MS technique: critical evaluation of the technique with 91500 zircon standard / Y. Orihashi [et. al.] // Journal of Mineralogical and Petrological Sciences. 2003. V. 98. P. 109-117.

6. Darling J.R., Storey C.D., Engi M. Allanite U-Th-Pb geochronology by laser ablation ICPMS // Chemical Geology. 2012. V. 292-293. P. 103-115.

7. Accurate U-Pb Age and Trace Element Determinations of Zircon by Laser Ablation-Inductively Coupled Plasma-Mass Spectrometry / Y. Honglin [et. al.] // Geostandards and Geoanalytical Research. 2004. V. 28, № 3. P. 353-370.

8. $\mathrm{Pb} / \mathrm{U}$ Fractionation during Nd:YAG $213 \mathrm{~nm}$ and $266 \mathrm{~nm}$ Laser Ablation Sampling with Inductively Coupled Plasma Mass Spectrometry / L. Haichen [et. al.] // Applied Spectroscopy. 2000. V. 54, № 10. P. 1435-1442.

9. Single zircon LA-ICPMS U-Pb dating of Weishan granite (Hunan, South China) and its petrogenetic significance / X. Ding [et. al.] // Science in China: Series D Earth Sciences. 2006. V. 49, № 8. P. 816-827.

10. U-Pb dating of zircon by LA-ICP-MS / Z. Chang [et. al.] // Electronic Journal of the Earth Sciences. 2006. V. 7, № 5. P. 1-14.

11. LA-MC-ICPMS and SHRIMP U-Pb dating of complex zircons from Quaternary tephras from the French Massif Central: Magma residence time and geochemical implications / A. Cocherie [et. al.] // Geochimica et. Cosmochimica Acta. 2009. V. 73. P. $1095-1108$.

12. High spatial resolution analysis of $\mathrm{Pb}$ and $\mathrm{U}$ isotopes for geochronology by laser ablation multi-collector inductively coupled plasma mass spectrometry (LA-MC-ICP-MS) / B. Buhn [et. al.] // An. Acad. Bras. Cienc. 2009. V. 81, № 1. P. 99-114.
13. Plesovice zircon - A new natural reference material for $U$ $\mathrm{Pb}$ and $\mathrm{Hf}$ isotopic microanalysis / J. Slama [et. al.] // Chemical Geology. 2008. V. 249. P. 1-35.

14. Jochum K.P., Stoll B. Reference materials for elemental and isotopic analysis. in: LA-ICP-MS in the Earth Sciences: Current Practices and Outstanding Issues // Ed. P.Sylvester. Mineralogical Association of Canada. 2008. V. 40. P. 147-168. 15. Black L.P., Gulson B.L. The age of the Mud Tank carbonatite, Strangways Range, Northern Territory // J.Aust. Geol. Geophys. 1978. V. 3. P. 227-232.

16. The application of laser ablation-inductively coupled plasma-mass spectrometry to in situ U-Pb zircon geochronology / S.E. Jackson [et. al.] // Chemical Geology. 2004. V. 211. P. 47-69.

17. Three Natural Zircon Standards for U-Th-Pb, Lu-Hf, TraceElement and REE Analyses / M. Wiedenbeck [et. al.] // Geostandards Newsletter. 1995. V. 19, № 1. P. 1-23.

18. Further Characterisation of the 91500 Zircon Crystal / M. Wiedenbeck [et. al.] // Geostandards and Geoanalytical Research. 2004. V. 28, № 1. P. 9-39.

19. Frei D., Gerdes A. Precise and accurate in situ U-Pb dating of zircon with high sample throughput by automated LASF-ICP-MS // Chemical Geology. 2009. V. 261. P. 261-270. 20. Precise determination of Phanerozoic zircon $\mathrm{Pb} / \mathrm{Pb}$ age by multicollector SIMS without external standardization / $\mathrm{Li}$ Xian-Hua [et. al.] // Geochemistry Geophysics Geosystems. 2009. V. 10, № 4. 21 p.

21. Zircon LA-ICP MS U-Pb Dating of the Longkang Andesitic Ignimbrites from Jiuzhaigou: Evidence of the Mianlue Suture Westward Extension / Q. Jiangfeng [et. al.] // Journal of China University of Geosciences. 2008. V. 19, № 1. P. 47-53. 22. Rosman K. J. R., Taylor P. D. P. Isotopic compositions of the elements 1997 (Technical Report) // Pure and Applied Chemistry. 1998. V. 70, № 1. Р. 217-235.

23. Пупышев А.А., Сермягин Б.А. Дискриминация ионов по массе при изотопном анализе методом масс-спектрометрии с индуктивно связанной плазмой // Екатеринбург: ГОУ ВПО УГТУ-УПИ. 2006. 132 с.

24. Messerly J. D. Current developments in laser ablation - inductively coupled plasma - mass spectrometry for use in geology, forensics, and nuclear nonproliferation research. PhD diss. lowa, 2008. 124 p.

25. Kooijman E., Berndt J., Mezger K. U-Pb dating of zircon by laser ablation ICP-MS: recent improvements and new insights // Eur. J. Mineral. 2012. V. 24. P. 5-21.

26. Alpha-decay event damage in zircon / T. Murakami [et. al.] // American Mineralogist. 1991. V. 76. V. 9-10. P. 1510-1532 27. Metamictisation of natural zircon accumulation versus thermal annealing of radioactivity-induced damage / L. Nasdala [et. al.] // Contrib. Mineral. Petrol. 2001. V. 141. P. 125-144. 28. Andersen T. Appendix A3: COMPBCORR - Software for common lead correction of $\mathrm{U}-\mathrm{Th}-\mathrm{Pb}$ analyses that do not report 204Pb. Mineralogical Association of Canada. 2008. V. 40. P. 1-18.

29. Цирконы и цирконовая геохронология гранитов Новоусмановского (Артлышского) массива (Южный Урал) / А.А. Краснобаев [и др.] // ИГ УНЦ РАН. Информационные материалы. Геологический сборник. № 10. 2013. С. 24-35.

\section{REFERENCES}

1. Zaitceva M.V., Pupyshev A.A., Shchapova J.V, Votyakov S.L. [Methodological aspects of $\mathrm{U} / \mathrm{Pb}$ dating of zircons using multicollector mass spectrometer with inductively coupled plasma NEPTUNE PLUS with NWR 213 attachment for laser ablation]. Analitika i kontrol' [Analytics and Control], 
2016, vol. 20, no. 2, pp. 121-137 (in Russian). doi: 10.15826/ analitika.2016.20.2.008

2. Longerich $\mathrm{H}$. Laser ablation-inductively coupled plasmamass spectrometry (LA-ICP-MS); An introduction. Mineralogical Association of Canada, 2008, vol. 40, pp. 1-18.

3. Vanhaecke F., Kyser K. The Isotopic Composition of the Elements. Degryse P.WILEY-VCH Verlag GmbH \& Co. KGaA, 2012, pp. 1-29.

4. Honglin Y., Fuyuan W., Shan G., Xiaoming L., Ping X., Deyou $\mathrm{S}$. Determination of $\mathrm{U}-\mathrm{Pb}$ age and rare earth element concentrations of zircons from Cenozoic intrusions in northeastern China by laser ablation ICP-MS. Chinese Science Bulletin, 2003, vol. 48, no. 22, pp. 2411-2421. doi: 10.1360/03wd0139 5. Orihashi Y., Hirata T., Tani K., Yoshida H. Rapid and simultaneous determination of multi-element abundances and $\mathrm{U}-\mathrm{Pb}$ age for zircon crystal using UV laser ablation ICP-MS technique: critical evaluation of the technique with 91500 zircon standard. Journal of Mineralogical and Petrological Sciences, 2003, vol. 98, pp. 109-117. doi: 10.2465/jmps.98.109 6. Darling J.R., Storey C.D., Engi M. Allanite U-Th-Pb geochronology by laser ablation ICPMS. Chemical Geology, 2012, vol. 292-293, pp. 103-115. doi: 10.1016/j.chemgeo.2011.11.012. 7. Honglin Y., Gao S., Liu X., Li H., Gunther D., Wu F. Accurate $\mathrm{U}-\mathrm{Pb}$ Age and Trace Element Determinations of Zircon by Laser Ablation-Inductively Coupled Plasma-Mass Spectrometry. Geostandards and Geoanalytical Research, 2004, vol. 28, no. 3, pp. 353-370. doi:10.1111/j.1751-908X.2004.tb00755.x 8. Haichen L., Borisov O., Mao X., Shuttleworth S., Russo R. $\mathrm{Pb} / \mathrm{U}$ Fractionation during Nd:YAG $213 \mathrm{~nm}$ and $266 \mathrm{~nm}$ Laser Ablation Sampling with Inductively Coupled Plasma Mass Spectrometry. Applied Spectroscopy, 2000, vol. 54, no. 10, pp. 1435-1442. doi:10.1366/0003702001948565

9. Ding X., Chen P., Chen W., Huang H., Zhou X. Single zircon LA-ICPMS U-Pb dating of Weishan granite (Hunan, South China) and its petrogenetic significance. Science in China: Series D Earth Sciences, 2006, vol. 49, no. 8, pp. 816-827. doi: 10.1007/s11430-006-0816-4

10. Chang Z., Vervoort J., McClelland W. C., Knaack C. U-Pb dating of zircon by LA-ICP-MS. Electronic Journal of the Earth Sciences, 2006, vol. 7, no. 5, pp. 1-14. doi: 10.1029/2005GC001100 11. Cocherie A., Fanning M., Jezequel P., Robert M. LA-MCICPMS and SHRIMP U-Pb dating of complex zircons from Quaternary tephras from the French Massif Central: Magma residence time and geochemical implications. Geochimica et. Cosmochimica Acta, 2009, vol. 73, pp. 1095-1108. doi:10.1016/j.gca.2008.11.028

12. Buhn B., Pimentel M.M., Matteini M., Dantas E. L. High spatial resolution analysis of $\mathrm{Pb}$ and $\mathrm{U}$ isotopes for geochronology by laser ablation multi-collector inductively coupled plasma mass spectrometry (LA-MC-ICP-MS). An. Acad. Bras. Cienc, 2009, vol. 81, no. 1, pp. 99-114. doi: 10.1590/S000137652009000100011

13. Slama J., Kosler J., Condor D.J., Crowley J.L., Gerdes A., Hanchar J.M., Horstwood S.A., Morris G.A., Nasdala L., Norberg N., Schaltegger U., Schoene B., Tubrett M.N., Whitehouse M.J. Plesovice zircon - A new natural reference material for $\mathrm{U}-\mathrm{Pb}$ and $\mathrm{Hf}$ isotopic microanalysis. Chemical Geology, 2008, vol. 249, pp. 1-35. doi: 10.1016/j.chemgeo.2007.11.005 14. Jochum K.P., Stoll B. Reference materials for elemental and isotopic analysis. In: $L A-I C P-M S$ in the Earth Sciences: Current Practices and Outstanding Issues. Mineralogical Association of Canada, 2008, vol. 40, pp. 147-168.

15. Black L.P., Gulson B.L. The age of the Mud Tank carbonatite, Strangways Range, Northern Territory. J.Aust. Geol. Geophys, 1978, vol. 3, pp. 227-232.
16. Jackson S.E., Norman J.P., William L.G., Belousova E.A. The application of laser ablation-inductively coupled plasma-mass spectrometry to in situ U-Pb zircon geochronology. Chemical Geology, 2004, vol. 211, pp. 47-69. doi: 10.1016/j. chemgeo.2004.06.017

17. Wiedenbeck M., Alle P., Corfu F., Griffin W.L., Meier M., Oberli F., Vonquadt A., Roddick, J.C., Speigel W. Three Natural Zircon Standards for U-Th-Pb, Lu-Hf, Trace-Element and REE Analyses. Geostandards Newsletter, 1995, vol. 19, no. 1, pp. 1-23. doi: 10.1111/j.1751-908X.1995.tb00147.x

18. Wiedenbeck M., Hanchar J.M., Peck W.H., Sylvester P., Valley J., Whitehouse M., Kronz A., Morishita Y., Nasdala L. Further Characterisation of the 91500 Zircon Crystal. Geostandards and Geoanalytical Research, 2004, vol. 28, no. 1, pp. 9-39. doi: 10.1111/j.1751-908X.2004.tb01041.x

19. Frei D., Gerdes A. Precise and accurate in situ U-Pb dating of zircon with high sample throughput by automated LASF-ICP-MS. Chemical Geology, 2009, vol. 261, pp. 261-270. doi: 10.1016/j.chemgeo.2008.07.025

20. Li X-H., Liu Y., Li Q-L., Guo Ch-H., Chamberlain K.R. Precise determination of Phanerozoic zircon $\mathrm{Pb} / \mathrm{Pb}$ age by multicollector SIMS without external standardization. Geochemistry Geophysics Geosystems, 2009, vol. 10, no. 4, 21 p.

21. Jiangfeng Q., Shaocong L., Guowei Z., Chunrong D., Yongfei L. Zircon LA-ICP MS U-Pb Dating of the Longkang Andesitic Ignimbrites from Jiuzhaigou: Evidence of the Mianlue Suture Westward Extension. Journal of China University of Geosciences, 2008, vol. 19, no. 1, pp. 47-53. doi: 10.1016/ S1002-0705(08)60023-0

22. Rosman K.J.R., Taylor P.D. P. Isotopic compositions of the elements 1997 (Technical Report). Pure and Applied Chemistry, 1998, vol. 70, no. 1, pp. 217-235. doi: 10.1063/1.556031 23. Pupyshev A.A., Sermiagin B.A. Diskriminatsiia ionov po masse pri izotopnom analize metodom mass-spektrometrii $s$ induktivno sviazannoi plazmoi [Discrimination ions according to mass at isotopic analysis by mass spectrometry with inductively coupled plasma]. Ekaterinburg, Ural State Technical University, 2006. 132 p. (in Russian).

24. Messerly J.D. Current developments in laser ablation - inductively coupled plasma - mass spectrometry for use in geology, forensics, and nuclear nonproliferation research. PhD diss. lowa, 2008. 124 p.

25. Kooijman E., Berndt J., Mezger K. U-Pb dating of zircon by laser ablation ICP-MS: recent improvements and new insights. Eur. J. Mineral, 2012, vol. 24, pp. 5-21. doi: 10.1127/09351221/2012/0024-2170

26. Murakami T., Chakoumakos B.C., Ewing R.C., Lumpkin G.R., Weber W.J. Alpha-decay event damage in zircon. American Mineralogist, 1991, vol. 76, no. 9-10, pp. 1510-1532 27. Nasdala L., Wenzel M., Vavra G., Irmer G., Wenzel T., Kober B. Metamictisation of natural zircon accumulation versus thermal annealing of radioactivity-induced damage. Contrib. Mineral. Petrol, 2001, vol. 141, pp. 125-144. doi: 10.1007/ s004100000235

28. Andersen T. Appendix A3: COMPBCORR - Software for common lead correction of $\mathrm{U}-\mathrm{Th}-\mathrm{Pb}$ analyses that do not report 204Pb. Mineralogical Association of Canada, 2008, vol. 40 , pp. 1-18.

29. Krasnobaev A.A., Puchkov V.N., Sergeeva N.D., Busharina S.V. [Zircon and geochronology of zircon granites Novousmanovskii (Artlyshskii) array (the South Urals)]. IG UNC RAN. Informacionnye materialy. Geologicheskij sbornik, 2013, no. 10, pp. 24-35 (in Russian). 